Service social

\title{
Un modèle développemental pour l'intervention auprès des familles recomposées
}

\section{Gerry Marino et Francine Fortier}

Volume 39, numéro 3, 1990

Familles recomposées après divorce

URI : https://id.erudit.org/iderudit/706504ar

DOI : https://doi.org/10.7202/706504ar

Aller au sommaire du numéro

Éditeur(s)

École de service social de l'Université Laval

ISSN

1708-1734 (numérique)

Découvrir la revue

Citer cet article

Marino, G. \& Fortier, F. (1990). Un modèle développemental pour l'intervention auprès des familles recomposées. Service social, 39(3), 112-134.

https://doi.org/10.7202/706504ar
Résumé de l'article

Sans une reconnaissance des caractéristiques de la famille recomposée, nous ne pouvons la traiter adéquatement.

Le présent article schématise le cheminement typique de ces familles à travers trois phases d'évolution. Il permet au praticien de cibler son intervention en conséquence et de distinguer ce qui est propre à la transition de ce qui renvoie à d'autres facteurs. 
Gerry Marino, psychologue, praticien en thérapie familiale au CLSC Lamater.

Francine Fortier, psychologue, pratique privée.

\title{
Un modèle développemental pour l'intervention auprès des familles recomposées
}

\author{
Gerry Marino \\ Francine Fortier
}

Le travail auprès d'une famille recomposée devrait s'appuyer sur un diagnostic différentiel dans le sens où, pour dégager des hypothèse valables, il faut savoir distinguer ce qui appartient à la recomposition de ce qui renvoie à d'autres facteurs. À défaut de faire cette distinction, le praticien risque de fonder son intervention sur de fausses prémisses. Pour fixer une bonne évaluation de situation, il devra donc s'exercer à discriminer trois catégories de problèmes :

- les difficultés normales et prévisibles qui sont liées à l'adaptation à la vie en famille recomposée;

- les problèmes qui sont liés au développement normal de l'enfant, de même qu'à la vie familiale en général;

- les problèmes associés à une psychopathologie ou à des problématiques particulières.

Cerner les difficultés qui sont propres à la recomposition n'est pas toujours une tâche évidente. Comme le phénomène est relativement nouveau, l'intervenant manque souvent d'outils pour le faire. Par ailleurs, la clientèle qui consulte ne représente pas nécessairement l'ensemble des familles de ce type. Le risque de généraliser les problèmes que la clientèle affiche et de voir la situation familiale comme en soi une source de dysfonctionnement s'en trouve accru. Le fait de mettre sur le compte de la recomposition des complications qui ne lui sont en rien attribuables limite la chance d'intervenir efficacement. 
Dans cette perspective, nous nous attarderons d'abord à dégager les éléments $d^{\prime}$ un cadre conceptuel servant autant à mettre en relief les spécificités de la famille recomposée qu'à situer notre modèle $d^{\prime}$ 'intervention. Cette première réflexion jettera les bases nécessaires à l'approfondissement de la notion de diagnostic différentiel. Dans la seconde partie, portant sur l'intervention et ses modalités, cette idée s'appliquera à une compréhension de plus en plus précise des problèmes courants et des complications, tantôt particulières et tantôt étrangères à la situation.

\section{Le cadre conceptuel}

\section{Perspective normalisante}

Carter et McGoldrick (1980) ont mis en évidence les multiples transitions meublant la vie d'une famille, soulignant que les difficultés tendent à surgir au cours de ces périodes cruciales nécessitant diverses adaptations. En parallèle, de plus en plus de chercheurs et de cliniciens reconnaissent l'existence d'une importante période de transition associée aux événements divorce et remariage (Ahrons et Rodgers, 1987; Cloutier, 1990; Felner et al., 1988; Hetherington et al., 1988; Visher et Visher, 1988). De fait, ces situations obligent la famille à des transformations structurelles et relationnelles profondes. Le modèle que nous proposons s'inscrit sur ces données fondamentales. Pour avoir une vision éclairée, le praticien devrait d'abord garder à I'esprit que toute famille recomposée traverse une période de transition pouvent s'étendre sur quelques années. Le passage demande une multitude d'adaptations et comporte des stress importants.

De ce point de vue, la recomposition s'apparente à d'autres circonstances auxquelles toute famille fait face. La naissance d'un premier enfant, son entrée à la maternelle, le départ de l'aîné de la famille sont autant de situations temporairement stressantes qui exigent des adaptations. Comme ces dernières nous sont familières, nous ne sommes pas portés à dramatiser les réactions qu'elles peuvent susciter. Ainsi, le petit qui s'accroche aux jupes de sa mère à sa première journée d'école, le jeune couple qui éprouve des difficultés à s'ajuster à son rôle de parents peuvent généralement compter sur l'appui et la compréhension de leur entourage. Tant que leurs difficultés apparaissent compréhensibles et acceptables, on anticipe avec confiance l'évolution et la stabilisation de la situation.

Toutefois, il n'y a pas toujours que des difficultés transitionnelles et prévisibles; de véritables problèmes peuvent également se développer. Ceux-ci se distinguent des embûches normales auxquelles on 
peut s'attendre. L'enfant atteint de phobie scolaire, la mère dont l'état dépressif nécessite une hospitalisation en sont des exemples. Le clinicien avisé n'attribuera pas ces phénomènes à la situation, mais plutôt à la vulnérabilité préalable de l'individu ou encore du système. En d'autres termes, il n'associera pas "entrée à l'école » ou « naissance d'un premier enfant " à " pathologie », mais croira que les circonstances ont favorisé l'apparition d'une organisation symptomatique.

Comme le divorce et la recomposition familiale sont des situations qui nous sont moins familières, nous avons malheureusement tendance à négliger ces distinctions essentielles et nous sommes portés à leur attribuer l'ensemble des problèmes constatés. Cette conception nous conduit à une vision pathologisante et stigmatisante de ces phénomènes. Pourtant, même s'ils éprouvent des difficultés passagères, des milliers d'adultes et d'enfants réussissent à s'adapter à leur nouvelle famille, et ce, sans développer de problèmes graves.

Dans notre perspective, il existe une spécificité propre à la famille recomposée. Il serait donc plus exact de considérer certaines difficultés comme prévisibles dans les circonstances. Par ailleurs, toute situation nouvelle met à l'épreuve les capacités d'adaptation de chacun. En ce sens, la recomposition peut parfois faire office d'événement précipitant. Étant donné la vulnérabilité de certains individus ou l'équilibre précaire de la famille précédente, il arrive que des symptômes fassent leur apparition. Reliés à des problèmes préexistants qui auraient pu surgir dans une autre situation stressante, ceux-ci ne doivent pas être confondus avec les difficultés transitionnelles courantes chez les familles recomposées.

La perspective normalisante n'a pas qu'un intérêt théorique, puisqu'elle s'avère aussi cruciale en ce qui concerne l'intervention. L'attitude du praticien à l'égard de la rupture et de la recomposition aura en effet une influence décisive sur l'efficacité de son travail. S'il perçoit ces événements d'un œil tragique plutôt que de les voir à l'origine d'une transition demandant certaines adaptations, il sera moins en mesure d'aider la famille à surmonter les défis qu'elle rencontre. Son intervention risque même de retarder ou d'empêcher l'adaptation.

\section{Perspective systémique}

Le second élément de notre cadre de référence renvoie à l'utilisation du langage et du modèle systémiques pour la compréhension de la famille recomposée et pour l'intervention. Parce que la nouvelle cellule regroupe plusieurs individus qui sont interreliés, la voir comme un système s'avère un outil précieux, voire indispensable. 
D'ailleurs, plus souvent qu'autrement, l'enfant participe à deux noyaux familiaux et la famille recomposée n'est en fait qu'un des sous-éléments d'un ensemble plus vaste comprenant plus d'une constellation familiale qui s'interinfluencent. Pour rendre compte de ces réalités, nous utiliserons tantôt le concept de famille binucléaire (Ahrons et Rodgers, 1987), tantôt celui de suprasystème (Sager et al., 1983).

Dans la perspective systémique, ce qui se passe dans un sousensemble, qu'il soit familial, dyadique ou individuel, ne peut être considéré isolément. Pour ne citer que quelques exemples se rapportant à la famille recomposée, la réaction de l'enfant au remariage de son parent influence le vécu du nouveau couple. L'attitude des adultes se répercute aussi sur l'enfant. Les conflits qui peuvent éclater entre lui et le beau-parent ont nécessairement un effet sur la relation conjugale et sur le lien filial. Au niveau du suprasystème, la famille recomposée est touchée, de diverses façons, par ce qui se passe dans l'autre maisonnée, et vice versa.

Ce qui se vit dans une partie du système a donc un impact sur l'ensemble des relations entre les multiples personnes en cause. Ces interinfluences tiennent de diverses considérations dont celles, par exemple, d'alliance, de hiérarchie et de frontière. Comme le mentionnent Visher et Visher (1988), la structure de la famille recomposée recèle aussi un potentiel élevé pour l'éclosion de coalitions et triangulations de tous genres. Développés par les théoriciens systémiques (voir, entre autres, Haley 1971, 1976, 1980 et Minuchin, 1974), tous ces concepts nous seront donc d'une grande utilité quand il s'agira d'élaborer un plan d'intervention approprié.

\section{Perspective développementale}

Attardons-nous maintenant à ce qui sous-tend essentiellement le modèle que nous proposons ici, soit le cheminement typique d'une famille recomposée. Le travail auprès de cette clientèle nous a amenés à le schématiser selon trois phases d'évolution, comprenant chacune une tâche développementale spécifique et des défis secondaires. Une fois surmontés les principaux problèmes du palier auquel elle se trouve, la famille est en mesure de faire face à ceux du suivant. L'évolution ne s'effectue pas toujours de manière absolument rigide, mais peut comporter un certain va-et-vient entre les différentes phases. Ainsi, ce qui n'a pas été complètement réglé pendant l'étape précédente peut être repris au stade suivant.

L'idée d'un cycle particulier à la famille recomposée n'est pas nouvelle. Adaptant, par exemple, le modèle présenté par Papernow 
(1984), Visher et Visher (1988) parlent de sept stades de développement menant à l'établissement d'une identité familiale solide, légitime et fonctionnelle. Tel que l'illustre le tableau préliminaire, cette période chapeaute les deux premières phases dans la typologie que nous proposons. Dans notre conception, la famille qui entre dans sa troisième phase s'est donc stabilisée, tandis que ses membres se sont ajustés à un nouveau mode de vie. Quant à la période d'établissement, nous l'avons qualifiée de phase transitionnelle, voulant rendre compte de deux réalités qui caractérisent la famille recomposée à ses débuts, soit la nécessité de se construire et celle de prendre en considération le passé qui la précède.

\section{TABLEAU PRÉLIMINAIRE}

\begin{tabular}{|l|l|l|l|}
\hline & \multicolumn{2}{|c|}{ Période de transition } & \\
\hline $\begin{array}{l}\text { Tâches tran- } \\
\text { sitionnelles }\end{array}$ & $\begin{array}{l}\text { - Réorganiser les liens et les } \\
\text { rôles } \\
\text { - S'adapter à sa nouvelle } \\
\text { situation de vie }\end{array}$ & \\
\hline Phases & Acceptation & Négociation & Élaboration \\
\hline $\begin{array}{l}\text { Tâches } \\
\text { développe- } \\
\text { mentales }\end{array}$ & $\begin{array}{l}\text { Accepter de } \\
\text { perdre et de } \\
\text { changer }\end{array}$ & $\begin{array}{l}\text { Établir un sys- } \\
\text { tème familial } \\
\text { viable }\end{array}$ & $\begin{array}{l}\text { Poursuivre } \\
\text { son devenir } \\
\text { familial }\end{array}$ \\
\hline
\end{tabular}

La naissance d'une famille recomposée présuppose une expérience d'éclatement familial, généralement suivie d'une réorganisation du système autour de deux noyaux séparés. À l'égard de ce passé, elle oblige d'abord un travail d'acceptation de la part de tous et chacun. De fait, l'enfant et l'autre parent se trouvent projetés dans une vie qu'ils n'ont pas choisie. Pour eux, il s'agit d'apprivoiser l'idée de l'existence d'un nouveau couple et celle de la nécessité d'une réorganisation conséquente. Mais avant de s'y faire, ils sont portés à défendre leurs intérêts. L'enfant, par exemple, tend à manœuvrer contre le nouveau couple, car il aspire encore à réconcilier ses géniteurs et est hostile à l'obligation de partager son parent. De leur côté, les époux aspirent à recréer instantanément la famille idéale et pensent que le beau-parent et l'enfant devraient s'aimer automatiquement. Les pressions qu'ils sont susceptibles d'exercer dans ce sens, de même que les conflits de loyauté éprouvés par le parent, ne font qu'alimenter les résistances des autres acteurs. Pour leur part, il s'agit donc de modifier leurs attentes, de regarder la réalité telle qu'elle se 
présente et de s'ouvrir à minimiser les pertes subies par l'enfant et l'autre parent.

La résolution de ces diverses questions permet l'entrée en phase de négociation. Plutôt que de remettre en question l'existence de la nouvelle cellule et d'en refuser les contraintes, on se préoccupe maintenant de se construire une place acceptable en son sein. Les arrangements du début sont peu à peu soumis à la critique, négociés puis remplacés par des solutions plus définitives parce que mieux adaptées au contexte de la famille recomposée. À ce stade, les confrontations doivent être considérées comme des efforts pour concilier les besoins individuels, conjugaux et familiaux qui se font compétition au sein du même système. Dans la mesure où l'on réussit cette tâche, on construit les bases nécessaires à la croissance et à la survie de la nouvelle famille. Avec la progression de ce travail, un sentiment d'appartenance émerge et l'identité familiale se dessine.

$S^{\prime}$ il lui reste à consolider ses acquis et à approfondir les nouveaux liens, la nouvelle cellule peut maintenant envisager un avenir commun réaliste, stable et acceptable pour tous. Au stade d'élaboration, elle ressemble à toute autre famille bien établie car elle a surmonté la majorité des bouleversements liés à la transition. Dès lors, son évolution sera surtout marquée par les grands événements du cycle de vie familiale. Elle aura à s'adapter aux nouvelles naissances, au passage de l'adolescence, au départ des enfants... en passant par les accidents de parcours et les aléas de la vie. En tout cela, elle conserve cependant son caractère distinct et l'élaboration se fait à partir des particularités inhérentes à sa structure. Exposée aux mêmes circonstances qu'une autre famille, elle aura sa propre façon de les traverser, son vécu s'étayant sur les possibilités offertes par sa constitution.

\section{L'intervention}

Comme il n'y a pas encore de tradition bien établie en matière de recomposition familiale, les gens qui la vivent sont facilement pris au piège de leurs difficultés. Ils sont encore plus vulnérables quand ils voient leur situation compliquée de problèmes qui n'ont pas à voir avec la transition actuelle ou qui sont carrément étrangers à la recomposition. Pensons seulement aux problèmes résiduels du passé, aux transitions liées au cycle de vie familiale, aux situations impliquant une triangulation ou une psychopathologie. C'est ici qu'intervient l'importance du diagnostic différentiel. Tandis que ses connaissances sur les particularités de la famille recomposée lui seront d'une utilité fort substantielle, le clinicien devra user de discernement pour établir son plan d'intervention. 


\section{La normalisation}

Face à toute famille en transition, la tâche essentielle de l'intervenant consiste à faciliter l'adaptation. L'atteinte de cet objectif passe par la normalisation. En ce qui concerne la recomposition familiale, les interventions à caractère normalisant sont d'autant plus indispensables qu'elles compensent le manque de tradition. À cet égard, l'une des fonctions importantes du praticien consiste à renseigner ses clients sur les difficultés courantes de la vie en famille recomposée. Cela se fait, bien sûr, à partir du matériel qu'on lui soumet. En ramenant peu à peu sur un registre normal les sentiments et les difficultés éprouvés par chacun, il travaille à ce que la famille comprenne mieux les particularités de sa situation et se sente moins marginale dans ce qu'elle vit. L'intervention de groupe est une formule qui, soit dit en passant, répond fort bien à ces sous-objectifs. Qu'il s'agisse de rencontres thématiques ou de réunions centrées sur le vécu des participants, le contexte permet, de surcroît, la reconnaissance mutuelle. L'impact normalisant ne peut que s'en trouver accru.

À elle seule, l'intervention éducative a de nombreux effets bénéfiques. En plus de servir à valider les émotions et les besoins respectifs, elle contribue à diminuer les inquiétudes des adultes tout en palliant leurs sentiments d'incompétence et de culpabilité. Enfin, elle favorise l'ajustement des attentes et des perceptions en vue d'une meilleure adaptation. À titre d'exemple, tous se verront soulagés d'apprendre l'universalité du rêve de réconciliation, de comprendre les résistances juvéniles ou d'entendre dénoncer les mythes de l'amour et de l'ajustement instantanés. En diminuant les pressions associées aux attentes irréalistes, ces révélations permettent à chacun de se réconcilier avec ses ambivalences tout en s'ouvrant aux sentiments d'autrui. Maintenant rassuré, le parent qui s'inquiétait de la réaction de ses protégés ou de l'attitude de son partenaire verra son rôle de façon plus objective et sera mieux équipé pour surmonter ses conflits de loyauté. L'enfant se sentira compris, tandis que son beauparent sera plus susceptible de s'armer de patience. La voie sera dégagée pour une clarification efficace des rôles et des frontières.

Dans ses efforts de normalisation, l'intervenant pourra aussi sensibiliser ses clients aux avantages inhérents à la recomposition familiale. De fait, par certains côtés, la famille recomposée ressemble à la famille élargie d'antan, ce qui lui confère des possibilités appréciables. Par exemple, à une époque où la famille a tendance à rétrécir, l'enfant qui vit dans ce cadre voit ses occasions de se lier multipliées. Pour leur part, les adultes peuvent avoir accès à un plus grand réseau d'entraide et jouissent parfois de congés parentaux réguliers. Sans minimiser les difficultés transitionnelles de ses clients, l'intervenant 
fait habilement entrevoir les gains relatifs à la nouvelle situation, ce qui contribue à modifier leur perception négative.

La normalisation passe également par la reconnaissance des difficultés reliées au développement de l'enfant. Par exemple, s'ils sont confrontés à la rébellion adolescente en même temps qu'ils ont à réorganiser la famille, parents et beaux-parents peuvent facilement confondre le défi éducatif avec les tâches liées à la transition familiale. Pareille confusion nuit à la recherche de moyens et d'attitudes adaptés aux besoins développementaux de l'enfant. Dans des circonstances comparables, l'intervenant doit s'attarder à parfaire les compétences parentales en renseignant ses clients sur les défis propres à chaque âge.

La normalisation peut aussi faire l'objet d'une intervention de réseau. Ici, le praticien s'adresse à différents acteurs ayant une influence sur le vécu de la famille, éducateurs, professeurs, entourage, autres intervenants... Lorsque cela est indiqué, il partage ses connaissances de la famille recomposée telles qu'elles s'appliquent à la cellule qui fait l'objet de la consultation. Mais sa tâche principale consiste à corriger les fausses perceptions et à dénoncer les préjugés nuisibles.

Bref, la normalisation est un outil fort valable qui peut s'appliquer sous différents modes d'intervention : consultation individuelle, conjugale, familiale, interventions de groupe et de réseau. Quand elle s'adresse à ceux qui vivent la situation, elle les aide à relever les défis rencontrés de façon plus appropriée. S'il est indispensable, l'outil a toutefois ses limites. C'est pourquoi il doit s'accompagner, dans plusieurs cas, d'une intervention à caractère thérapeutique.

\section{L'intervention thérapeutique ciblée}

Toute famille recomposée est susceptible de voir son évolution compromise par l'une ou l'autre des difficultés propres à sa situation. Quand l'impasse se dessine, le praticien devra s'attarder à la dénouer. C'est là l'objectif principal de l'intervention thérapeutique ciblée.

Comme la recomposition familiale est ponctuée de stades comportant chacun ses défis, l'intervention sera fort différente selon l'étape à laquelle est rendue la famille. Prenons l'exemple d'une consultation concernant des conflits aigus entre membres de deux fratries. Voyant que les enfants n'ont pas encore accepté le remariage de leur parent, le clinicien abordera le comportement qui fait problème comme une résistance à la recomposition. Si, par contre, les mêmes conflits se tissent sur la trame de la deuxième phase, il devra considérer qu'ils servent plutôt à la négociation d'un espace relationnel ou d'un territoire physique. La démarche thérapeutique passe donc 
d'abord par la détermination du niveau développemental de la famille. En élaborant les considérations relatives à chacun des stades, nous préciserons la nature des cibles d'intervention alors appropriées. Pour un survol rapide, le lecteur pourra aussi consulter le tableau synthèse présenté à la fin de l'article.

\section{L'intervention au stade d'acceptation}

Parmi les motifs fréquents de consultation, on retrouve ici les plaintes et les questionnements concernant l'attitude hostile des enfants ou du beau-parent. Derrière le discours officiel se cachent généralement d'importantes déceptions conjugales et familiales. La sous-estimation des difficultés d'adaptation relatives à la relation beau-parent-enfant et la méconnaissance des véritables motifs derrière l'attitude de l'enfant favorisent l'escalade des conflits. La combinaison des attitudes antithétiques des adultes et des enfants est parfois explosive, surtout avec l'adolescent qui peut voir mille raisons de se rebeller.

Alors que l'enfant a surtout besoin qu'on reconnaisse ses pertes et qu'on soit ouvert à les minimiser, l'incompréhension mutuelle s'entretient et contamine les relations. Les sentiments de perte et les attentes irréalistes seront donc les premières cibles d'intervention. Le praticien dénoncera les mésattributions nuisibles en prêtant une intention positive à tous ceux qui sont concernés. Le mythe de l'amour instantané sera contré par une vision plus réaliste et les pertes seront reconnues. En même temps, on soulignera la nécessité de s'adapter et de s'ouvrir à négocier.

Au-delà de cette première manche, un travail de consolidation du couple est souvent indiqué. Encore fragile et vulnérable en comparaison des liens antécédents, le système couple risque d'être emporté par les vents et marées des premières tempêtes. Ultimement, l'intervention visera l'affirmation de la primauté du couple au sein de la nouvelle unité familiale. Pour ce faire, les frustrations et les conflits de loyauté gagneront à être reconnus et surmontés. L'intervenant amènera ensuite les époux à renouveler leur engagement de manière plus réaliste, tout en les incitant à être fermes, vis-à-vis des enfants, quant à l'impossibilité d'un retour en arrière.

Tout ce travail favorisera l'entrée en phase de négociation. Une fois leurs perceptions nuisibles dénoncées et leur couple consolidé, les époux seront mieux équipés pour travailler le système à d'autres niveaux. Quant aux enfants, tant que leur parent était aux prises avec ses conflits de loyauté, ils pouvaient espérer un retour en arrière, mais devant la fermeté actuelle de l'engagement, ils n'ont d'autres choix que de se résigner. Dès lors, ils chercheront la place qui leur 
revient au sein de la nouvelle famille et, bientôt, ils forceront les adultes à clarifier leurs rôles et leurs statuts.

\section{L'intervention au stade de négociation}

Au stade de négociation, la famille a pour tâche de se doter d'un fonctionnement satisfaisant, propre à assurer sa viabilité. Les conflits entre les divers membres de la cellule constituent les principaux motifs de consultation. Ils devront être accueillis comme le signe d'un désir d'engagement plus profond.

Des deux époux, c'est généralement le beau-parent qui amorce le mouvement vers le changement. Une fois le couple consolidé, sa volonté de participation ne laisse plus de place aux accommodations plus ou moins timorées des premiers temps. Tandis que l'explosion des émotions, la difficulté à communiquer ou l'intolérance des différences contribuent à envenimer les différends, on vient alors consulter pour savoir qui a tort ou raison. II peut s'agir de différences dans les styles éducatifs, de désaccord en ce qui concerne les règlements de la maison, de divergences d'opinion sur les méthodes à appliquer avec tel ou tel enfant...

En phase de négociation, pareils désaccords sont inévitables. Même quand on veut le taire ou le nier, l'antagonisme entre les époux favorise la persistance ou l'apparition de conflits entre l'enfant et le beau-parent. Comme le rôle et le statut parental du dernier reste à clarifier, le premier est susceptible de chercher avantage. Pourquoi, en effet, voudrait-il se soumettre si son parent ne l'oblige pas à le faire ? Par ailleurs, la rivalité entre membres des deux fratries est, elle aussi, inéluctable. Souvent, elle est l'expression d'une saine compétition pour déterminer une distribution plus équitable de l'affection et de l'attention parentales. La chambre à coucher, les effets personnels, le contrôle des aires de la maison peuvent également devenir les champs de bataille où se négocient les questions de rang, de territoire et de pouvoir.

Généralement plus explosifs qu'auparavant, les conflits avec l'autre maisonnée complètent le tableau. Parfois, on se plaint d'avoir à subir une ingérence révoltante de l'autre parent; parfois, on cherche à lui imposer sans succès ses propres valeurs et façons de faire. Ici, la conjoncture conflictuelle incite l'enfant à manipuler les adultes de part et d'autre.

Au stade de négociation, le rôle de l'intervenant consiste à aider ses clients à se détacher de l'objet de leurs mésententes pour s'intéresser aux questions plus fondamentales qu'elles suscitent. Pour être viable, la famille doit maintenant se définir un modus vivendi qui, tout en étant fonctionnel, soit approprié aux besoins respectifs de ses 
membres. On pourra souligner que toute famille novice a à négocier les options qui s'offrent à elle et que les conflits à ce sujet ne sont point réservés aux cellules recomposées. Cela étant dit, la démarche thérapeutique passe d'abord par la reconnaissance des besoins individuels et conjugaux des adultes et par la reconnaissance des besoins affectifs, éducatifs et développementaux des enfants. Le praticien enclenchera ensuite le processus de négociation en favorisant la recherche de compromis équitables et l'invention de solutions originales et adaptées. En tout cela, il encouragera la tolérance des différences et l'efficacité de la communication.

Par ailleurs, pour devenir fonctionnelle, la famille doit travailler à restaurer la frontière intergénérationnelle, ébranlée par les circonstances des premiers temps. C'est pourquoi, dans la plupart des cas, le système parent doit devenir la cible privilégiée de l'intervention. On motivera les époux à devenir solidaires dans leurs efforts pour maîtriser la situation. Tantôt, on encouragera la participation du parent, tantôt, on légitimera celle du beau-parent, l'un et l'autre ayant à négocier valeurs, méthodes éducatives et règlements de la maisonnée. On les aidera aussi à clarifier leurs rôles et statuts respectifs en tenant compte des limites imposées par l'âge des enfants. Tout cela débouchera sur le rétablissement de la hiérarchie parents-enfants à l'intérieur de la cellule immédiate, un gain qui sera profitable aux derniers autant qu'aux premiers.

Lorsqu'il y a des problèmes persistants au niveau suprasystémique, il faut également travailler la frontière entre les deux maisonnées. La famille binucléaire comporte en effet des exigences qui devront être reconnues. Elle ne permet à aucun des systèmes parents de détenir le pouvoir auquel nous a habitués la famille intacte. Que I'un ou l'autre abuse en s'ingérant dans les affaires de l'autre, et c'est le choc. Cette susceptibilité impose la levée d'une frontière claire entre les deux milieux. Cependant, celle-ci doit rester assez perméable pour permettre à l'enfant de voyager à l'aise entre ses deux foyers. Selon les circonstances, le clinicien aidera donc le couple à accepter les limites de son pouvoir et de ses responsabilités ou à en définir les possibilités. II encouragera aussi la négociation d'ententes et de rapports mutuellement satisfaisants avec les adultes de I'autre maisonnée.

\section{L'intervention au stade d'élaboration}

Au stade d'élaboration, la famille est moins susceptible de venir consulter, car elle a surmonté la majorité des bouleversements liés à la transition. Toutefois, il n'est pas rare que le passage de l'adolescence suscite des remises en question assez importantes pour pous- 
ser le couple à chercher de l'aide. Ici, le praticien peut être porté à croire que les difficultés sont l'expression d'une mauvaise adaptation à la recomposition. La nature des problèmes tend d'ailleurs à renforcer cette impression : un départ fracassant pour aller vivre chez l'autre parent, l'apparition ou la recrudescence de la rébellion à l'égard du beau-parent, la dénonciation du divorce, l'expression d'une souffrance attribuée à la recomposition... À première vue, ces problèmes ressemblent étrangement aux difficultés des premiers temps.

Toutefois, si nous examinons la situation de plus près, il devient évident que ces manifestations se rattachent plutôt aux thèmes universels de l'adolescence. Lorsqu'il traverse le passage, l'enfant élevé dans une famille recomposée exprime ses conflits personnels et son tumulte intérieur dans des termes permis par le contexte. Ainsi, la fugue chez l'autre parent est un moyen tout désigné pour s'affirmer contre l'autorité parentale. Porté à remettre en question sa famille, l'adolescent trouve aussi une cible de prédilection en la personne de son beau-parent. L'éclatement de sa famille d'origine pourra être évoquée comme la cause de tous ses malheurs ou devenir l'excuse universelle pour ses écarts de conduite. En famille recomposée, ce genre d'énoncés est susceptible de remplacer les fantaisies traditionnelles de l'adoption cachée et de l'enfant non désiré.

Lorsqu'une famille bien établie rencontre des problèmes avec un adolescent, l'intervenant prendra garde de ne pas devenir complice $d^{\prime}$ une remise en question de la famille. Sachant que les touches paranoïdes et dépressives d'une jeunesse en herbe s'étayent ici sur un fond de recomposition, il saura que l'enjeu véritable est la mise au monde d'un jeune adulte. II lui faudra plutôt renseigner les parents sur les défis éducatifs liés à l'adolescence tout en s'intéressant à la problématique sous-jacente. La plupart du temps, il s'agit de conflits relatifs à l'affranchissement, à la quête de l'identité ou à la lutte pour I'autonomie.

Ainsi, on consulte souvent parce que l'enfant réclame un changement de formule de garde, tandis qu'on n'y est pas préparé. Pour l'adolescent de famille binucléaire, le départ pour l'autre maisonnée peut être une façon de prendre ses distances par rapport au milieu qui l'a principalement élevé, dans le but légitime de compléter son identité. Toutefois, il peut aussi s'agir d'une menace, en réaction à des conflits au sujet de la discipline et du contrôle. Pour le contrer adéquatement, il faudra donc décider laquelle des problématiques est en cause. La première demande la révision des arrangements de garde et l'acceptation d'une perte, soit pour l'enfant, soit pour les adultes. L'autre exige plutôt un travail du système parents-enfant, comparable à celui qu'on entreprendrait dans n'importe quelle famille. 
Par ailleurs, il arrive aussi que le comportement de I'adolescent force la collaboration entre les deux sous-systèmes familiaux. Certains entreprennent, par exemple, une véritable carrière d'enfant prodigue en effectuant des aller-retour entre leurs deux foyers. Tandis que ce manège leur sert à échapper à l'autorité parentale, une concertation entre les deux milieux est indispensable pour mettre fin à l'échappatoire. Au stade d'élaboration, les frontières sont suffisamment claires pour qu'un travail du suprasystème ne porte pas à la confusion comme ce serait le cas dans les premiers temps de la recomposition.

Le passage de l'adolescence nous a servi à illustrer comment les événements du cycle de vie familiale sont vécus différemment dans une famille recomposée. Pour éviter de contribuer à la confusion, le clinicien devra les aborder comme des manifestations universelles qui s'expriment selon le caractère unique de la famille.

\section{Les complications}

Par ailleurs, la famille recomposée peut aussi rencontrer des problèmes particuliers qui demandent une intervention appropriée. Au contraire des difficultés précédentes, qu'on peut associer à un stade particulier, ces problèmes sont susceptibles de se manifester à n'importe quel moment du développement. Ils imposent alors des stress supplémentaires à la famille, ce qui a pour effet de ralentir et de complexifier le processus évolutif. Ces complications ne sont pas universelles, c'est-à-dire qu'on ne les rencontre pas dans chaque famille recomposée. Toutefois, la plupart des familles qui viennent consulter en connaissent une, sinon plusieurs.

Lorsque le praticien rencontre des complications, il doit d'abord s'attaquer à la problématique présentée. À notre avis, son intervention doit cependant tenir compte du fait que la famille est une famille recomposée et du stade où elle se trouve dans son évolution. L'exposé des complications les plus fréquentes mettra en relief ces considérations.

\section{La triangulation}

Sous la coupe de leur impuissance, les adultes des deux maisonnées peuvent mettre l'enfant au centre des problèmes qu'ils ne parviennent pas à résoudre. Il peut s'agir de difficultés reliées aux transformations suscitées par le remariage ou bien de problèmes résiduels associés au divorce. L'enfant est souvent volontaire pour occuper pareille position. Tandis qu'on le charge d'un rôle idiosyncratique, il devient porteur d'un fardeau trop lourd pour lui, car il ne peut résoudre un problème que seuls les adultes sont en mesure de régler. 
L'enfant témoin en est un premier exemple : pris à témoin des fautes commises, des injustices subies, des humiliations vécues, on l'érige en juge et on lui confie la tâche de décider qui est coupable. Proche cousin, l'enfant messager devient la cible de la frustration et de l'agressivité adressée à l'expéditeur, tandis qu'on le charge de transmettre les revendications, reproches, récriminations ou insatisfactions. Autre instance fréquente de triangulation, l'enfant otage devient le pion dont on se sert pour marquer des points et gagner la bataille : c'est celui qu'on laisse sur le pas de la porte quand maman n'est pas au rendez-vous, qu'on envoie en haillons pour que l'autre fasse sa part, qu'on prive d'une sortie avec papa pour se venger d'une faute réelle ou imaginée. Dans toutes ces triangulations, on retrouve souvent le fameux triangle du persécuteur, de la victime et du sauveteur.

Le nouveau couple est également susceptible de trianguler l'enfant. II peut, par exemple, faire peser le poids de l'insatisfaction conjugale sur les épaules du petit ou se convaincre que son attitude peu conciliante est la cause du dysfonctionnement familial. Pris au centre de conflits non avoués entre les conjoints, l'enfant terrible se trouve confirmé dans son sentiment de culpabilité envers l'éclatement originel. Si, par la suite, le nouveau couple devait échouer, l'effet risquerait d'être dévastateur.

Quand il identifie une triangulation mettant l'enfant en cause, I'intervenant tentera d'abord de réhabiliter les conjoints dans leur pouvoir de négocier et dans leur capacité à dénouer le conflit. Une clarification du problème et des enjeux le situera là où une solution peut être apportée, c'est-à-dire entre adultes. Un autre niveau d'intervention vise l'enfant plus directement. On le soulagera de sa culpabilité en dénonçant l'omnipuissance et la pensée magique qui entretiennent sa croyance qu'à lui seul il peut dissoudre le couple actuel ou réunir ses parents. On lui dira clairement que les adultes ne s'attendent pas à ce qu'il trouve une solution à leur place et que ce n'est pas à lui de déterminer qui a tort ou raison.

Dans le contexte d'une recomposition, certains adultes peuvent également être triangulés. Si son partenaire adopte la position de victime tandis que l'ancien conjoint occupe celle de persécuteur, le beau-parent peut s'interposer pour le défendre. Pour sa part, le parent peut être quelque peu étriqué entre les exigences déraisonnables et contradictoires posées par son ex-conjoint et par son nouveau partenaire. Cette dernière instance de triangulation est relativement fréquente. Dans les situations de triangulation entre adultes, l'intervention visera l'abandon des rôles idiosyncratiques chez ceux qui viennent consulter. Dès qu'un des participants au triangle vicieux change d'attitude, le jeu ne peut plus continuer. 


\section{Les coalitions}

À ses débuts, la famille recomposée réunit tous les ingrédients nécessaires pour favoriser une coalition intergénérationnelle contre le beau-parent. D'abord, à côté de l'alliance naturelle entre le parent et sa progéniture, l'alliance conjugale n'est pas encore édifiée. Aux prises avec des sentiments de culpabilité à l'égard de ses protégés, le parent devient vite la proie de ses conflits de loyauté. Ajoutez à cela un beau-parent qui, tôt ou tard, fait mentir le mythe de l'amour instantané en manifestant son agressivité et sa frustration, et le résultat est souvent le suivant : un parent et son enfant se liguent, tacitement ou plus ouvertement, contre un méchant beau-parent. Si le vent de l'ambivalence souffle toujours, la lutte se termine fréquemment par le renvoi du conjoint ou par son découragement.

À titre d'illustration, citons l'exemple d'une dame qui consulte au sujet des luttes intenses qui sévissent entre elle et l'adolescente de son mari. Satisfaite de sa relation conjugale, elle menace toutefois de quitter son nouveau foyer si les conflits avec sa belle-fille ne se résorbent pas. L'absence du père et de sa fille à la première consultation est, à elle seule, révélatrice. L'exploration de la situation démontre d'ailleurs que les conflits sont polarisés entre belle-mère et belle-fille tandis que le père forme, avec la dernière, une coalition par omission. Par moments, celle-ci devient plus active : Monsieur fait des reproches à Madame, l'incitant à trouver un terrain d'entente avec sa fille rebelle et lui suggérant de venir consulter. Dans les situations où il y a pareille polarisation, il est souhaitable de rendre la personne périphérique plus centrale. Dans le cas qui nous intéresse, le couple a été convoqué à l'entrevue suivante, ce qui nous a permis d'encourager le père à faire équipe avec sa femme et à jouer un rôle plus actif face aux comportements rebelles de sa fille.

Une autre forme de coalition intergénérationnelle est celle qui unit l'enfant et l'autre parent contre le nouveau couple. C'est le cas, par exemple, de celui qui encourage son adolescent à contester les exigences du beau-parent ou encore à s'opposer à l'autorité parentale de l'autre foyer. Ici, l'enfant est susceptible d'utiliser à son avantage le contexte difficile d'une famille binucléaire en redéfinition. Même quand elle se fonde sur des exigences jugées déraisonnables, la coalition ne peut qu'être nocive en ce qu'elle envenimera nécessairement les relations. Évidemment, l'intervention variera selon la position de ceux qui viennent consulter. Face au parent qui se ligue avec l'enfant, on soulignera les dangers de ne point respecter les frontières et l'autonomie de l'autre milieu. On encouragera aussi l'enfant à régler ses différends avec les adultes concernés. Quant à ceux qui sont victimes de la coalition, ils pourront douter d'eux et leur ambivalence minera leur autorité. En explorant les possibilités de manipulation par l'en- 
fant, on renforcera le système parent et la frontière entre les deux maisonnées. Si le couple est rigide, il viendra plutôt consulter dans l'espoir de reprendre le contrôle de la situation, mais il aura davantage besoin de s'ouvrir à la négociation. Dans tous les cas, c'est au couple visé par la coalition qu'il appartient de trouver un terrain d'entente avec l'enfant.

Tantôt complémentaire à la coalition citée précédemment, tantôt indépendante, I'alliance entre nouveaux partenaires peut s'établir au détriment de l'ex-conjoint. Quasi normal dans les débuts, ce phénomène est susceptible de tourner en coalition. C'est le cas, par exemple, du couple qui cherche à éliminer les contacts de l'enfant avec leur encombrant partenaire de fortune. Pareille attitude rend impossible la mise en place d'ententes fonctionnelles régissant les rapports entre les deux maisonnées. Ici, il faudra faire comprendre aux époux que, nonobstant leurs raisons, la coalition n'est ni à leur avantage, ni à celui de l'enfant. Une fois reconnu l'intérêt à respecter les droits de visite, on encouragera les époux à lever une frontière adéquate entre les deux foyers, ce qui aura pour effet d'abaisser les risques de récidives.

Bien que moins fréquente, la coalition entre ex-conjoints est une chose possible. Après la rupture et le remariage, rester alliés dans l'exercice de la fonction parentale est en soi positif. Toutefois, quand I'alliance devient coalition, elle ne peut qu'être nuisible pour la nouvelle cellule car elle empêche l'intégration du nouveau conjoint. Quand on prend, sans le consulter, des décisions ayant des conséquences pour lui, ce dernier finira par se révolter. Dans de telles circonstances, l'intervenant sensibilisera le parent à l'importance de développer une loyauté envers son nouveau partenaire. S'il y a lieu, il soulignera aussi les dangers d'utiliser l'ex-conjoint dans la négociation d'une relation avec lui.

\section{Le syndrome de l'enfant de famille éclatée}

Par «syndrome de l'enfant de famille éclatée » nous voulons désigner une gamme de problèmes qui sont en fait le vestige de difficultés d'adaptation postdivorce ou encore de carences reliées au dysfonctionnement conjugal et familial antérieur. Du point de vue étiologique, on peut associer ce syndrome au principe de périodes critiques pour l'apprentissage. Au cours du développement, certains moments sont plus favorables que d'autres à telle ou telle acquisition. Passé la période critique, l'enfant a plus de difficulté à les intégrer. Pensons seulement à l'apprentissage de la propreté, à l'intériorisation de l'interdit, à l'acquisition d'une certaine confiance en soi, pour n'en nommer que quelques-unes. $\mathrm{Si}$, aux prises avec leurs propres dilem- 
mes, les parents n'ont pas pu assurer, au moment opportun, l'encadrement et la sécurité nécessaires à faire progresser l'enfant, certains retards développementaux peuvent se glisser. Pareille conjoncture n'est pas l'apanage des foyers brisés, mais il n'en demeure pas moins que la dysharmonie familiale conduisant à la rupture ou encore les stress de l'adaptation postdivorce la favorisent.

Les carences dans les apprentissages de base sont inévitablement reportées dans la nouvelle famille et représentent un défi supplémentaire pour les conjoints. En effet, on ne peut s'attendre à ce que l'enfant énurétique, immature, impulsif ou dépendant corrige ses lacunes instantanément. Pour le faire progresser, il faudra plutôt appliquer les méthodes éducatives appropriées à la petite enfance. Temps, efforts, constance et encadrement seront nécessaires. L'explication de l'origine et de la nature de ces difficultés aide les nouveaux époux à surmonter leur frustration et à s'armer de patience et de persévérance. Plutôt que de continuer à accuser le passé ou à rechercher un coupable, ils se tournent alors vers l'avenir, voyant la recomposition comme une occasion nouvelle de corriger la situation.

Encore une fois, un bon diagnostic différentiel permet de situer le problème à un niveau approprié à la recherche de solutions pertinentes. II faut savoir démêler les problèmes antécédents des réactions normales et passagères à la recomposition. Bien qu'il puisse être momentanément accentué par les circonstances des premiers temps, le retard dans les apprentissages de base n'est pas relié à la recomposition puisqu'il était installé bien avant l'événement remariage. On ne peut donc le qualifier de réactionnel et on doit s'attendre à ce qu'il persiste même si les stress du début sont dissipés. Par ailleurs, il ne faut pas généraliser, certains problèmes préexistants renvoyant à une tout autre étiologie. Pensons seulement à I'autisme ou à I'hyperactivité, soupçonnés d'avoir un fondement biologique.

\section{Les problèmes associés à une psychopathologie}

Plusieurs familles consultent aussi pour des problèmes reliés à la psychopathologie d'une personne appartenant à la cellule immédiate ou au suprasystème. Lorsqu'une pareille situation fait l'objet de la demande d'aide, la pathologie deviendra la cible privilégiée de l'intervention. Il peut s'agir d'une psychose, d'un trouble de personnalité, d'un problème de violence familiale ou de toxicomanie, etc. Comme ce sont là des problèmes très complexes, on ne peut pas toujours les résoudre définitivement. Toutefois, l'intervenant se doit de circonscrire le problème pour que, au moins, il ne fasse plus obstacle à la transition. En cela, il lui faut tenir compte du contexte de la recomposition. Ainsi, sa connaissance du cheminement normal de 
la famille recomposée l'aidera à mieux planifier son intervention. Par exemple, dans le choix d'une tâche thérapeutique à la phase d'acceptation, sa préférence ira vers le parent biologique pour l'attribution du rôle central. Afin de savoir qui engager dans la thérapie, il devra s'éclairer des mêmes considérations développementales.

Pour appuyer notre propos, citons l'exemple d'un couple qui consulte au sujet d'une mésentente profonde en ce qui a trait au comportement de I'adolescent de Madame : Monsieur trouve que son épouse n'est pas assez sévère, tandis que sa partenaire déplore les explosions fréquentes entre son fils et son mari. S'il s'agit d'un motif de consultation typique à la transition, l'exploration révèle toutefois que le jeune homme est impliqué dans le monde de la criminalité et de la drogue, tandis que père, mère et beau-père n'ont que peu de contrôle sur lui. C'est pourquoi le diagnostic différentiel désigne la délinquance du garçon comme première cible d'intervention. Tant que les difficultés de cette tierce personne n'étaient pas reconnues, les conjoints étaient réduits à des sentiments d'impuissance et de frustration qu'ils imputaient à tort à leur situation familiale. Par surcroît, leurs sentiments contradictoires et leurs problèmes de restructuration avaient pour effet de réduire leur efficacité d'éducateur. L'intervention a consisté principalement à mettre en relief la sociopathie du garçon et à la distinguer des difficultés reliées à la transition familiale. Il a fallu encourager le couple à faire équipe et mobiliser la mère à prendre des dispositions plus efficaces par rapport à la tendance de son garçon. Comme le système parents était encore chambranlant, et puisque les frontières entre les deux foyers restaient à définir, il n'aurait pas été judicieux $d^{\prime}$ inviter le père à participer aux séances thérapeutiques.

Par ailleurs, comme psychopathologie et problèmes relationnels vont généralement de pair, les personnes souffrant de troubles psychologiques sont susceptibles de vivre un éclatement familial. Par le biais, il arrive qu'une famille recomposée connaisse des difficultés relatives à une pathologie chez l'autre parent. Ici, le problème de fond est encore plus difficile à régler, car ce n'est point la personne atteinte qui formule la demande. Ainsi, un certain aménagement de la situation est souvent le seul objectif réaliste que nous puissions nous fixer.

À ce titre d'illustration, parlons d'une famille qui, recomposée depuis quatre ans, implique une belle-mère sans enfant avec un père et ses filles de 9 et 10 ans. L'éclatement de la première famille avait suivi I'hospitalisation temporaire de la mère en psychiatrie. Le couple consulte au sujet de la cadette. II la trouve trop renfermée et craint qu'elle ne reste marquée par les mauvais traitements infligés par la mère. Effectivement, avant son hospitalisation, celle-ci traitait ses fil- 
les sur un modèle de clivage, l'une étant la "bonne", l'autre la " mauvaise ": tandis qu'elle louangeait sans cesse l'aînée, elle confinait la plus jeune à la cuisine, prétextant qu'elle était la fille du démon. Les nouveaux conjoints se demandent principalement comment réagir face aux droits de visite de la mère qui, depuis peu, réclame sa "bonne fille " à l'exclusion de la "mauvaise ". Le père et la belle-mère ont d'abord été appuyés dans leur idée de ne pas exposer la petite à une recrudescence des mauvais traitements. Pareille intervention visait la protection de l'enfant dans les limites qu'offrait la situation. Un second niveau d'intervention s'adressait directement aux fillettes, victimes de la pathologie de leur mère. L'une se sentait responsable du rejet et craignait de susciter un nouvel éclatement. L'autre se culpabilisait d'être préférée et se sentait déchirée entre sa mère et sa sœur. En présentant la pathologie de la mère comme la source de ces conflits intérieurs et en voyant la nouvelle famille comme un lieu sûr et légitime pour la réparation et pour la gratification des besoins, on a soulagé les enfants d'une bonne part de leurs angoisses. Bien établie, la famille a pu poursuivre son cheminement sans se laisser contaminer par les difficultés occasionnées par la pathologie de la mère.

\section{Symptomatologie au service du rêve de réconciliation}

L'enfant qui développe un symptôme dans le contexte de sa famille recomposée pose un piège aux parents et intervenants. Lorsque le comportement est alarmant, il n'est pas rare que les ex-conjoints se mobilisent dans l'espoir d'aider leur enfant. L'intervenant a souvent le même réflexe, croyant bien faire en sollicitant le concours de l'ancien couple. Or, pareille façon de faire contribue plutôt au maintien du symptôme, surtout si, par la même occasion, on éloigne le beauparent. Le problème est alors encouragé à persister car, en concrétisant le fantasme de réconciliation, il acquiert une fonction secondaire qui finit par remplacer la première motivation. Tout cela est plus ou moins conscient chez l'enfant.

Pour toutes ces considérations, le clinicien avisé évitera de convoquer l'ancienne famille en entrevue, se concentrant plutôt sur la nouvelle. II s'attaquera au symptôme là où il se présente, c'est-à-dire dans le foyer qui fait appel à ses services. Quant aux adultes de l'autre maisonnée, ils pourront, s'il y a lieu, être mis à contribution, mais à condition qu'on apporte une attention particulière au respect des frontières. Ainsi, si l'une ou l'autre des familles est dans sa première phase, on lui donnera un rôle accessoire et on évitera, surtout, de confronter les deux maisonnées. Si, par contre, les deux sont bien établies, le travail peut impliquer la collaboration des deux systèmes. 


\section{Les incongruences des cycles de vie}

Dans un couple remarié il est fréquent que l'un des conjoints n'ait pas eu l'occasion d'avoir ses propres enfants. S'il lui en vient le désir, il risque de trouver à ses côtés un partenaire repu qui souhaite plutôt consacrer ailleurs ses énergies. Pareille incompatibilité des besoins impose un stress additionnel à la relation. Souvent, les désirs contradictoires sont minimisés, voire niés. Ils s'expriment alors indirectement et peuvent donner lieu à divers motifs de consultation camouflant une insatisfaction difficile à avouer.

Le rôle de l'intervenant consiste alors à clarifier les désirs sousjacents et à soutenir les conjoints dans la révision nécessaire de leur choix. L'évaluation des options à la lumière du véritable problème deviendra le centre de l'intervention. Il faudra soupeser l'intensité des désirs incompatibles et la mettre en relation avec l'importance accordée au projet conjugal. S'ils souhaitent poursuivre leur vie à deux, les époux devront déterminer lequel des désirs rivaux primera. Au pire, le travail débouchera sur une rupture consentie, qui aura au moins l'avantage d'avoir été réfléchie.

La jeune femme qui se lie à un homme plus âgé connaîtra, elle aussi, des difficultés imputables à une incongruence des cycles de vie. Son inexpérience de la vie conjugale et familiale la rendra particulièrement vulnérable aux stress transitionnels occasionnés par la recomposition. On n'a qu'à songer au statut ambigu de celle qui se trouve projetée dans une famille où les adolescents ne sont guère plus jeunes qu'elle. Ici, la hiérarchie belle-mère-enfants est pratiquement impossible à installer. C'est pourquoi l'intervenant découragera les tentatives pour reproduire la relation maternelle traditionnelle. Il apportera une attention particulière à la solidité des frontières entourant le couple. Dans des circonstances où la différence d'âge est assez marquée pour justifier la clarification des rôles parentaux, le travail consistera surtout à éduquer et à normaliser. Moins la bellemère est expérimentée, plus elle aura besoin d'appui et d'encouragement. Des considérations comparables pourraient s'appliquer aux situations mettant en scène un beau-père jeune ou inexpérimenté.

\section{Conclusion}

La recomposition familiale est un phénomène de plus en plus répandu, bien qu'elle soulève diverses difficultés. Pour répondre efficacement à la demande croissante venant de ce type de clientèle, il apparaît fondamental de bien connaître les particularités de sa situation. De fait, la compréhension de la famille recomposée ne peut être 


\section{TABLEAU SYNTHÈSE}

\begin{tabular}{|c|c|c|}
\hline PHASE D'ACCEPTATION & PHASE DE NÉGOCIATION & PHASE D'ÉLABORATION \\
\hline $\begin{array}{l}\text { Tâches développementales } \\
\text { Accepter les pertes et le changement }\end{array}$ & Établir un système familial viable & Poursuivre le devenir familial \\
\hline $\begin{array}{l}\text { Difficultés prévisibles } \\
\text { - Effets réciproques de l'attitude des } \\
\text { adultes et de celle des enfants } \\
\text { - Difficultés d'adaptation. } \\
\text { - Hostilité ou rivalité entre l'enfant } \\
\text { et le beau-parent } \\
\text { - Conflits de loyauté } \\
\text { - Alliances intergénérationnelles } \\
\text { - Arrangements interfamiliaux non } \\
\text { satisfaisants } \\
\text { - Lune de miel écourtée }\end{array}$ & $\begin{array}{l}\text { - Explosion des émotions et com- } \\
\text { munication plus ou moins fruc- } \\
\text { tueuse } \\
\text { - Intolérance des différences et dif- } \\
\text { ficulté à faire des compromis } \\
\text { - Conflits entre les conjoints, entre } \\
\text { les enfants de deux fratries, entre } \\
\text { les deux sous-systèmes familiaux }\end{array}$ & $\begin{array}{l}\text { - Remise en question suscitée } \\
\text { par le passage d'un enfant à } \\
\text { l'adolescence }\end{array}$ \\
\hline $\begin{array}{l}\text { Cibles d'intervention } \\
\text { - Attentes irréalistes } \\
\text { - Sentiments de perte } \\
\text { - Ambivalences : conflits de loyauté } \\
\text { et frustrations } \\
\text { - Consolidation du système couple }\end{array}$ & $\begin{array}{l}\text { - Définition des besoins } \\
\text { - Communication et recherche de } \\
\text { compromis } \\
\text { - Renforcement du système parent } \\
\text { - Frontière entre les deux maison- } \\
\text { nées }\end{array}$ & $\begin{array}{l}\text { - Problématique adolescente : } \\
\text { identité, affranchissement, } \\
\text { négociation de l'autonomie } \\
\text { - Collaboration entre les deux } \\
\text { maisonnées }\end{array}$ \\
\hline \multicolumn{3}{|l|}{ Complications les plus fréquentes } \\
\hline \multicolumn{3}{|c|}{ Triangulation, coalition, symptomatologie au service du rêve de réconciliation } \\
\hline \multicolumn{3}{|c|}{ Syndrome de l'enfant de famille éclatée } \\
\hline \multicolumn{3}{|c|}{ Problèmes associés à une psychopathologie } \\
\hline & Incongruence des cycles de vie & \\
\hline
\end{tabular}


assimilée à celle de la famille intacte. Sans une connaissance de ce qui constitue la norme pour ce type de famille, nous ne pouvons prétendre intervenir efficacement. Cernant le cheminement qu'on doit considérer comme normal pour une famille recomposée, la perspective développementale fournit une base plus réaliste pour l'évaluation et pour l'intervention. Effectivement, elle précise les difficultés inhérentes au processus de recomposition, ce qui empêche de les confondre avec un dysfonctionnement. Sans ce pivot théorique, nous ne pouvons nous départir de nos préjugés nuisibles, non plus qu'élaborer un plan d'intervention approprié. Surtout, nous ne sommes pas aptes à susciter, chez nos clients, les attitudes et comportements propices à faciliter la transition ou le succès global de leur entreprise.

Le modèle que nous proposons s'insère dans cette perspective en ce qu'il met en relief les enjeux et les défis de la vie en famille recomposée. Toutefois, il prône la nécessité de prendre en considération le niveau développemental de la famille, tant pour l'interprétation de ses difficultés que pour le choix d'un plan d'intervention. Là réside son apport particulier. Trop souvent, on attribue à la famille recomposée un statut de précarité permanente. Pareille conception ne peut que nourrir la confusion du couple qui rencontre des problèmes au stade d'élaboration. Encore plus fréquemment, on focalise sur les questions de deuil au détriment des nécessités d'adaptation. Or, la famille recomposée n'a pas que deuils à résoudre. Une intervention qui se limite aux pertes subies par les enfants ou aux attentes irréalistes entretenues par les adultes est loin d'être suffisante. On ne doit pas oublier que la famille a à se construire, et que de la qualité du bâtiment dépend sa permanence. Bref, il ne faut pas escamoter les enjeux de la négociation et il faut prendre en considération les préoccupations et les besoins du nouveau couple. Notre modèle veut tenir compte de ces réalités qui nous apparaissent cruciales quand il s'agit de promouvoir la survie du regroupement. En déterminant les points tournants dans la vie d'une famille recomposée, il permet à l'intervenant de situer ses clients et de cibler son travail en conséquence.

\section{Références bibliographiques}

Ahrons, C.R. et R.H. Rodgers (1987). Divorced Families : Meeting the Challenge of Divorce and Remarriage. New York : W.W. Norton \& Company.

CARTer, E.A. et M. MCGoldrick (1980). A Family Life Cycle : A Framework for Family Therapy. New York : Gardner Press.

Cloutier, R. (1990). Avant-propos, dans Apprentissage et socialisation, vol. 13, $\mathrm{n}^{\circ} 1: 3-6$. Spécial jeunes et nouvelles familles. 
FELNER, R.D., L. TeRRE et R.T. Rowlison (1988). A Life Framework for Understanding Marital Dissolution and Family Reorganisation, dans S.A. WoLCHIK et P. KAROLY, Children of Divorce : Empirical Perspectives on Adjustment. New York et Londres : Gardner Press.

Haley, J. (1971). Changing Families : A Family Therapy Reader. New York: Grune et Stratton.

Haley, J. (1976). Problem Solving Therapy: New Strategies for Effective Family Therapy. San Francisco : Jossey-Bass.

Haley, J. (1980). Leaving Home. New York : McGraw-Hill.

Hetherington, E.M., J.D. Arnett et E.A. Hollier (1988). « Adjustment of Parents and Children to Remarriage" ", dans Children of Divorce, op. cit.

MinUCHIN, S. (1974). Families and Family Therapy. Cambridge : Harvard University Press.

PAPERNOW, P. (1984). "The Stepfamily Life Cycle : An Experiential Model of Stepfamily Development ", Family Relations, vol. 33 : 355-363.

Sager, C.J., H.S. Brown, H. Crohn, T. Encel, E. Rodstein et L. Walker (1983). Treating Remarried Family. New York : Brunner-Mazel.

VISHER, E.B. et J.S. VISHER (1988). Old Loyalties, New Ties : Therapeutic Strategies with Stepfamilies. New York : Brunner-Mazel. 